\title{
Hystrix primigenia (WAGNER, 1848) (RODENTIA, MAMMALIA) DEL MIOCENO SUPERIOR (MN13) DE LAS CASIONES (FOSA DE TERUEL, ESPAÑA)
}

\author{
Luis ALCALÁ' y Plinio MONTOYA² \\ ' Museo Nacional de Ciencias Naturales (CSIC). José Gutiérrez Abascal 2, \\ 28006 Madrid, España. \\ - Departament de Geologia, Universitat de València. Doctor Moliner 50, \\ $461(0)$ Burjassot, España.
}

Alcalá, L. y Montoya, P. 1998. Hystrix primigenia (Wagner, 1848) (Rodentia, Mammalia) del Mioceno Superior (MN13) de Las Casiones (Fosa de Teruel, España). [Hystrix primigenia (Wagner, 1848) (Rodentia, Mammalia) from the Upper Miocene (MN13) of Las Casiones (Teruel Basin, Spain)]. Revista Española de Paleontología, 13 (2), 139-147. ISSN 0213-6937.

\begin{abstract}
The porcupine remains from the Upper Miocene (Upper Turolian, MN13) of Las Casiones, province of Teruel, Spain, are described. Due to the large size of the dentition and its relatively brachyodont aspect, they are included in the species Hystrix primigenia (Wagner, 1848). Porcupine fossils from Las Casiones are the most abundant and the best represented of this species in Spain. In the present study, the material from Venta del Moro (also MN13), formerly adscribed to Hystrix sp., is now classified as $H$. primigenia. All these data are consistent with the hypothesis that $H$. primigenia arrived in the Iberian Peninsula during the late Turolian and remained there, at least, up to the late Pliocene (Villarroya, La Rioja), which represents one of the latest appearances of this species in the fossil record.
\end{abstract}

Keywords: Hystrix, Rodentia, Mammalia, Upper Miocene, Upper Turolian, Las Casiones, Teruel Basin, Spain.

\section{RESUMEN}

Se estudian los restos de puercoespines procedentes del yacimiento del Mioceno Superior (Turoliense superior, MN13) de Las Casiones, en la provincia de Teruel, España. Se han identificado como pertenecientes a Hystrix. primigenia (Whagner, 1848), en función de la gran talia y del carácter relativamente braquiodonto de la dentición. Los fósiles de Las Casiones constituyen el conjunto más abundante y mejor representado de restos de dicha especie en España. En el presente trabajo se determina asimismo el material de Hystrix sp. de Venta del Moro (también de la MN13) como H. primigenia. Todo esto se halla en consonancia con la hipótesis de la entrada de H. primigenia durante el Turoliense superior en la Península Ibérica, donde permanece al menos hasta el Plioceno Superior (Villarroya, La Rioja); esta última es una de las citas más modernas en el registro fósil de la especie.

Palabras clave: Hystrix, Rodentia, Mammalia, Mioceno Superior, Turoliense superior, Las Casiones, Fosa de Teruel, España.

\section{INTRODUCCIÓN}

El yacimiento de vertebrados de Las Casiones se encuentra en la cabecera del barranco del mismo nombre, localizado entre las poblaciones turolenses de Celadas y Villalba Baja, a cuyo término municipal pertenece (Fig. 1). Se sitúa en una serie de niveles lacustres del Mioceno Superior, compuestos fundamentalmente por arcillas verdes y negras, con intercalaciones ocasionales de arenas finas. En ellos se ha obtenido una abundantísima fauna de mamíferos de características coincidentes con las de la localidad clásica de El Arquillo, de edad Turoliense superior (zona MN 13). Este conjunto se ubica en el contexto de una serie carbonatada que se interpreta como perteneciente a depósitos lacustres muy someros, eventualmente desecados o anegados en ocasiones por depósitos de llanura fangosa.

Durante 1993 y 1994, se llevaron a cabo excavaciones sistemáticas según el método expuesto en Alcalá (1994). En el transcurso de los trabajos, se excavó una capa de 


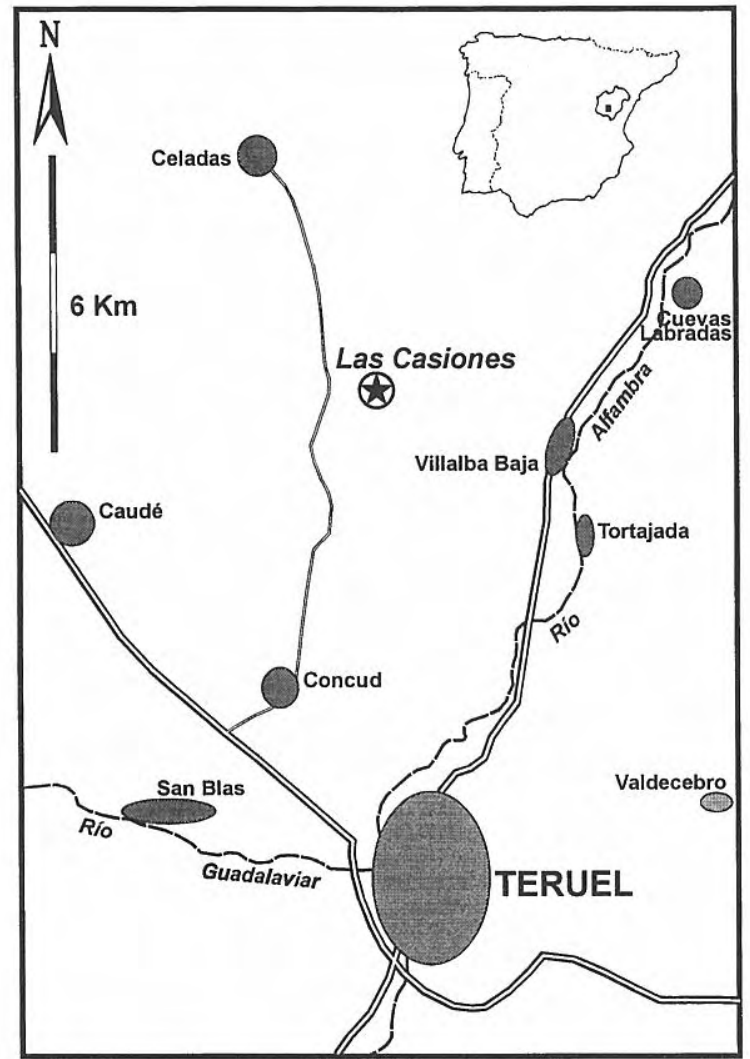

Figura 1. Situación geográfica del yacimiento de Las Casiones.

arcillas negras y otra, inmediatamente debajo, compuesta por arcillas verdes; los fósiles de Hystrix aquí estudiados provienen de ambos niveles.

La macrofauna determinada en Las Casiones está compuesta mayoritariamente por équidos del género Hipparion, el macromamífero más frecuente en el Mioceno de la Fosa de Teruel. Abundan especialmente los restos de las especies más pequeñas del género: Hipparion gromovae Villalta y Crusafont, 1957 e Hipparion periafricanum Villalta y Crusafont, 1957. Esta última es muy poco frecuente en el registro fósil, considerándose una especie endémica de España. Los artiodáctilos están bien representados por un bóvido de gran talla del género Tragoportax y, en mucha menor cantidad, por elementos pertenecientes a cérvidos. Aunque muy escaso, es destacable el registro de un hipopótamo. Los carnívoros, como es habitual en este tipo de asociaciones, presentan proporciones bajas, aunque se han encontrado interesantes restos de mustélidos y del gran úrsido Agriotherium. La asociación de macromamíferos conocida hasta el momento consta de: Agriotherium sp., Plesiogulo monspessulanus Viret, 1939, Mustela sp., Baranogale adroveri Petter, 1964, Mustelidae indet., Sabadellictis sp., Thalassictis hipparionum Gervais, 1850 ex Nordmann, Paramachairodus aff. orientalis (Kittl, 1887), Amphimachairodus giganteus (Wagner, 1848), Felidae indet., Hexaprotodon crusafonti (Aguirre, 1963), Tragoportax sp., Boselaphini indet., Pliocervus aff. matheroni (Gervais, 1859),
Pliocervus turolensis Azanza, 1989, Hipparion primigenium (Meyer, 1829), Hipparion gromovae Villalta y Crusafont, 1957, Hipparion periafricanum Villalta y Crusafont, 1957, Lartetotherium schleiermacheri (Kaup, 1834), Pliohyrax cf. graecus (Gaudry, 1862), Tetralophodon longirostris (Kaup, 1835) y Zygolophodon turicensis (Schinz, 1883). Información acerca de la fauna puede encontrarse en Cerdeño (1989; 1990), Alcalá (1992; 1994) y Alcalá et al. (1994). La semejanza de esta asociación de macromamíferos con la del yacimiento de El Arquillo (presencia de hipopótamo, de las dos especies de Hipparion de pequeña talla, del mismo Boselaphini grande, etc.) permite atribuir a Las Casiones la misma edad, Turoliense superior. Además, los micromamíferos indican una edad correspondiente a la parte media (zona M2) del Turoliense superior (van Dam et al., en preparación).

En cuanto a los elementos anatómicos más frecuentemente registrados, hay que señalar la abundancia de dientes aislados, que abarcan más de la mitad del total de restos identificados. Asimismo, destaca la proporción relativamente alta de elementos del autópodo, representados fundamentalmente por huesos del carpo y del tarso. En cuanto a los demás componentes, se registran presencias minoritarias de elementos del esqueleto axial, de las cinturas, del estilópodo y del zeugópodo, todos ellos generalmente fragmentarios. Esta representación de restos óseos permite proponer la existencia de procesos de alteración diferencial durante la etapa bioestratinómica, mediante concentración de los elementos esqueléticos más resistentes a la meteorización.

En este sentido, no es de extrañar que los ejemplares de Hystrix recuperados sean restos dentarios, mandibulares y maxilares, sin que se hayan reconocido elementos del esqueleto postcraneal. Con todo, se trata del conjunto más abundante y mejor representado de fósiles de dicha especie en la Península Ibérica.

\section{SISTEMÁTICA}

ORDEN RODENTIA Bowdich, 1821

Familia Hystricidae Burnet, 1830

Género Hystrix Linneo, 1758

\section{Hystrix primigenia (Wagner, 1848)}

Fig. 2, Lám. I

Sinonimias: en Sulimski (1960) y van Weers (1994).

Localidad tipo: Pikermi (Grecia), Turoliense medio (MN12).

Otras localidades: Alifakas, Dytiko 3, Halmyropotamos y Samos (Grecia), Kalimanci (Bulgaria), Umen Dol (Yugoslavia), Weze (Polonia), Serrat d'en Vacquer (Francia), Brisighella (Italia), Kuruksai (Tadzikistan), Taraklia (Bessarabia), Çoban Pinar y Kemiklitepe (Turquía), Menacer (Argelia), Las Casiones, Layna, Venta del Moro, 
Villalba Alta, Villarroya y Villastar (España) y, posiblemente, en Polgardi (Hungría).

\section{Material}

Los ejemplares procedentes del yacimiento de Las Casiones se hallan depositados en el Museo Nacional de Ciencias Naturales de Madrid (MNCN) y en el Museo Paleontológico de la Universidad de Zaragoza (MPZ).

MNCN KS-7: serie superior derecha $\mathrm{P}^{4}-\mathrm{M}^{3}$

MNCN KS-1: $\mathrm{M}^{1-2}$ izquierdo

MPZ-96/226 K93-1078: M $^{1-2}$ derecho

MNCN KS-52: molar superior derecho indeterminado

MNCN KS-54: molar superior derecho indeterminado

MNCN KS-122: hemimandíbula izquierda con $\mathrm{P}_{4}-\mathrm{M}_{3}$

MPZ-96/225 K93-1003: M $1-2_{2}$ izquierdo

\section{Descripción}

Para la descripción de los dientes se ha seguido la nomenclatura propuesta por van Weers (1990, 1993); las dimensiones se han tomado según el método utilizado por van Weers $(1990,1993)$ y van Weers y Montoya (1996).

\section{Serie superior $\mathrm{P}^{4}-\mathrm{M}^{3}$ (KS-7) (Lám. I, 7):}

Los cuatro dientes muestran un contorno casi circular y un avanzado estado de desgaste, que es mayor en el $\mathrm{M}^{1} \mathrm{y}$ menor en el $\mathrm{P}^{4}$. El $\mathrm{P}^{4}$ presenta cinco islas de esmalte y los pliegues labiales II y III aún ligeramente abiertos, y por tanto un estado de desgaste E-F. En cambio, ninguno de los tres molares mantiene pliegues abiertos, hallándose el $\mathrm{M}^{1}$ en un estado de desgaste $\mathrm{H}$ y el $\mathrm{M}^{2}$ y $\mathrm{M}^{3}$ en un estado $\mathrm{G}$.

\section{M ${ }^{1-2}$ (KS-1, K93-1078) (Fig. 2, a; Lám. I, 8-11):}

Los dos ejemplares son más largos que anchos. Ambos presentan facetas de contacto tanto anterior como posterior; por ello, los consideramos como $\mathrm{M}^{1-2}$. Son dos dientes con idéntica morfología, igual estado de desgaste (grado C) y de lados contrarios, por lo que con toda probabilidad pertenencen a un mismo individuo. Muestran un patrón morfológico constituido por tres pliegues labiales abiertos (I, II y III), quedando bifurcada la apertura del pliegue III debido a la presencia de una pequeña columna de esmalte. Además, en ambos dientes se produce la unión del pliegue lingual (VI) con el pliegue labial II.

Molares superiores indeterminados (KS-52, KS-54):

El primero de ellos se encuentra algo deteriorado, por lo que no se observa bien la morfología oclusal; es un diente más largo que ancho que, al parecer, posee dos o tres pliegues labiales abiertos y al menos una isla de esmalte formada, hallándose probablemente en un estado de desgaste C. El segundo de los ejemplares reseñados es también más largo que ancho y está muy poco desgastado (clase A-B).

\section{Hemimandíbula con $\mathrm{P}_{4}-\mathrm{M}_{3}$ (KS-122) (Lám. I, 1-3):}

El hueso mandibular se halla muy deformado por aplastamiento y, por tanto, poco se puede decir acerca de su morfología, excepto que el borde anterior de la fosa masetérica se sitúa debajo del $\mathrm{M}_{1}$. Los dientes son claramente más largos que anchos y presentan un estado de

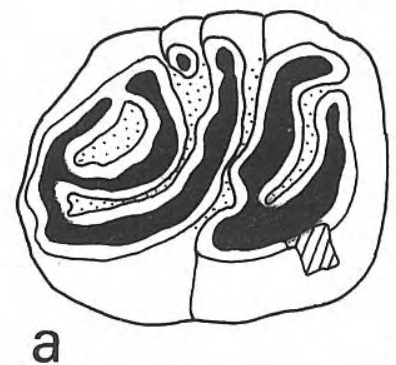

$5 \mathrm{~mm}$

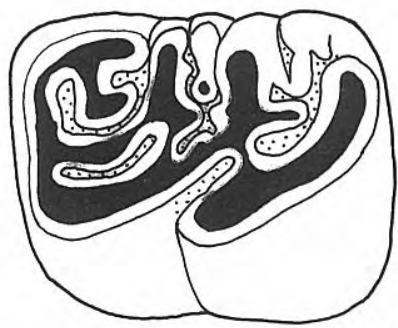

b

Figura 2. Molares de Hystrix primigenia (Wagner, 1848) de Las Casiones. a) $\mathrm{M}^{1-2}$ derecho (MPZ-96/226 K93-1078); b) $\mathrm{M}_{1-2}$ izquierdo (MPZ-96/225 K931003).

desgaste medio-alto. Al igual que ocurría con la serie superior, el $M_{1}$ es el diente más desgastado, mientras que el $\mathrm{P}_{4}$ es el que menos abrasión muestra. Todos los dientes presentan dos pliegues linguales abiertos así como un número variado de islas de esmalte: 4 en $\mathrm{P}_{4}, \mathrm{M}_{1}$ y $\mathrm{M}_{2}$, mientras que el $M_{3}$ posee hasta 7. Los cuatro dientes parecen encontrarse en un estado de desgaste R-S.

M 1 (K93-1003) (Fig. 2, b; Lám. I, 4-6):

Diente claramente más largo que ancho, con facetas de contacto anterior y posterior; por tanto, debe de tratarse de un $\mathrm{M}_{1 \cdot 2}$. Posee cuatro pliegues linguales abiertos y una isla de esmalte. Este ejemplar presenta un menor grado de desgaste que los dientes de la hemimandíbula, estimándose un estado Q.

\section{Discusión}

Los patrones de morfología oclusal de la dentición se muestran muy conservadores en toda la familia Hystricidae; esto, unido a la considerable variación que dichos patrones experimentan conforme el diente se desgasta, hace a veces difícil utilizar criterios morfológicos para la distinción entre especies (ver van Weers, 1990, 1994; van Weers y Montoya, 1996). De todos modos, aspectos tales como la gran talla y el carácter relativamente braquiodonto de la dentición aquí estudiada (Tabla 1, Fig. 3) ofrecen pocas dudas acerca de la inclusión del puercoespín de Las Casiones en Hystrix primigenia (Wagner, 1848), especie que se puede considerar típica del Mioceno Superior y Plioceno en el área circunmediterránea. Así, los dientes descritos en el presente trabajo son mucho mayores que los de $H$. parvae (Kretzoi, 1951) (Fig. 3) y claramente más braquiodontos que los de $H$. refossa (Gervais, 1852), que son las dos especies de puercoespines con las que, al parecer, llega a solaparse cronológicamente.

El límite inferior del rango bioestratigráfico de $H$. primigenia no está muy claro, ya que se registra con seguridad en el Turoliense medio (MN12) de Pikermi (Wagner, 1848; Gaudry, 1862-1867) y de Kemiklitepe (Sen, 1994), pero quizás su registro sea más antiguo. En este sentido, la especie ha sido citada en Halmyropotamos (de Bonis et al., 1992), yacimiento al que se 


\begin{tabular}{|ll|c|c|c|c|c|}
\cline { 3 - 7 } \multicolumn{1}{l|}{} & $\begin{array}{c}\text { Clase } \\
\text { de } \\
\text { desgaste }\end{array}$ & $\begin{array}{c}\text { Longitud } \\
\text { superficie } \\
\text { oclusal }\end{array}$ & $\begin{array}{c}\text { Anchura } \\
\text { superficie } \\
\text { oclusal }\end{array}$ & $\begin{array}{c}\text { Altura } \\
\text { corona } \\
\text { (Cr. ht. })\end{array}$ & $\begin{array}{c}\text { Altura } \\
\text { esmalte } \\
\text { (En. ht.) }\end{array}$ \\
\hline KS-7 & $\mathrm{P}^{4}$ & $\mathrm{E}-\mathrm{F}$ & 12,6 & 13 & 8,7 & 10,9 \\
& $\mathrm{M}^{1}$ & $\mathrm{H}$ & 10,3 & 12,9 & & 5,9 \\
& $\mathrm{M}^{2}$ & $\mathrm{G}$ & 11,1 & & 9 & 7,8 \\
& $\mathrm{M}^{3}$ & $\mathrm{G}$ & 10,9 & 10,1 & 8 & 9,2 \\
\hline $\mathrm{KS}$-1 & $\mathrm{M}^{1-2}$ & $\mathrm{C}$ & 11,6 & 8,5 & $10 \mathrm{ca}$ & $13,5 \mathrm{ca}$ \\
\hline $\mathrm{K} 93-1078$ & $\mathrm{M}^{1-2}$ & $\mathrm{C}$ & 10,9 & 8,7 & 10 & 13,5 \\
\hline $\mathrm{KS}-52$ & $\mathrm{M}^{\text {sup }}$ & $\mathrm{C} ?$ & 10,9 & 8,5 & & $15 \mathrm{ca}$ \\
\hline $\mathrm{KS}$-54 & $\mathrm{M}^{\text {sup }}$ & $\mathrm{A}-\mathrm{B}$ & 9,5 & 7,4 & 12,3 & 17 \\
\hline $\mathrm{KS}$-122 & $\mathrm{P}_{4}$ & $\mathrm{R}-\mathrm{S}$ & 11,3 & 8,6 & & 8,1 \\
& $\mathrm{M}_{1}$ & $\mathrm{R}-\mathrm{S}$ & 10,5 & 8,5 & & 5,7 \\
& $\mathrm{M}_{2}$ & $\mathrm{R}-\mathrm{S}$ & 11,5 & 9,4 & & 6,4 \\
& $\mathrm{M}_{3}$ & $\mathrm{R}-\mathrm{S}$ & 10,8 & 8,9 & & 6,1 \\
\hline K93-1003 & $\mathrm{M}_{1-2}$ & $\mathrm{Q}$ & 11,7 & 8,3 & 10,2 & 7,7 \\
\hline
\end{tabular}

Tabla 1. Dimensiones en $\mathrm{mm}$ de los dientes yugales de Hystrix primigenia (Wagner, 1848) de Las Casiones (ca: cálculo aproximado). La clase de desgaste según van Weers (1990). La medida Cr. ht. (altura de la corona) se ha tomado en la muralla posterior, en los dientes superiores, y en la muralla lingual, en los dientes inferiores; la medida En. ht. (altura del esmalte) se ha tomado en la muralla lingual, en ambos casos (ver van Weers, 1990, 1993; van Weers y Montoya, 1996).

atribuye una edad Turoliense inferior (MN11). Recientemente, van Weers (1994) argumenta que Hystrix trofimovi (Shevyreva, 1986), del Plioceno Superior de Tadzikistan, debe ser sinónimo de $H$. primigenia; elevándose así el límite superior de su rango, aunque ya se había citado la especie en Villarroya (Agustí et al., 1987), yacimiento perteneciente a la MN16 (de Bruijn et al., 1992).

Desde un punto de vista biogeográfico, podemos decir que $H$. primigenia muestra una amplia distribución en el área circunmediterránea, extendiéndose por Europa, oeste de Asia y norte de Africa. Así, en el Turoliense, se halla presente en los yacimientos griegos de Halmyropotamos, Samos, Pikermi, Alifakas y Dytiko 3 (Wagner, 1848; Gaudry, 1862-1867; Solounias, 1981; de Bonis et al., 1992), en Çoban Pinar y Kemiklitepe (Turquía) (Ozansoy, 1965; Sen, 1994), en Kalimanci (Bulgaria) (Sen y Kovatchev, 1987), en Umen Dol (Yugoslavia) (Garevski, 1956), en Taraklia (Bessarabia) (ver van
Weers, 1994), en Brisighella (Italia) (Masini y Rook, 1993), en Menacer (Argelia) (Arambourg, 1959; Thomas y Petter, 1986; Montoya, 1993), muy probablemente en Polgardi (Hungría) (ver van Weers, 1994), así como en los yacimientos españoles de Venta del Moro (Morales, 1984), Villastar (Mein et al., 1990; Adrover et al., 1993) y en el aquí estudiado de Las Casiones.

En el Plioceno, $H$. primigenia ha sido citado en Serrat d'en Vacquer (Francia) (Depéret, 1890-1892), Weze (Polonia) (Sulimski, 1960), Kuruksai (Tadzikistan) (Nikiforowa y Vangengeim, 1988; van Weers, 1994), y en España en Villalba Alta (Adrover, 1986), Layna y Villarroya (Agustí, et al., 1987).

\section{EL GÉNERO Hystrix EN ESPAÑA}

En el registro fósil de la Península Ibérica se encuentran representadas las principales formas del género Hystrix del Neógeno (incluido el Cuaternario) europeo (ver Fig. 4).

El registro más antiguo de Hystrix en España corresponde a una forma de pequeña talla procedente del Turoliense inferior (MN11) de Crevillente 2, originalmente asignada a la especie Hystrix suevica por Montoya (1993). Con posterioridad, el holotipo de $H$. suevica de Schlosser (1884) fue invalidado, e incluido en el género Anchitheriomys, por van Weers (1993). Recientemente, van Weers y Montoya (1996) han llevado a cabo la revisión de las formas pequeñas de Hystrix encontradas en unos pocos yacimientos europeos del Vallesiense superior, como son Csákvár (Kretzoi, 1951) y Kohfidisch (Bachmayer y Wilson, 1970) y del Turoliense inferior, como Salmendingen (Schlosser, 1902) y Crevillente 2 (Montoya, 1993, 1994). Van Weers y Montoya (1996) reúnen estas formas bajo la denominación de Hystrix parvae (Kretzoi, 1951), que representa la especie más antigua del género. $H$. parvae se caracteriza por su pequeña talla, dentición braquiodonta y la presencia frecuente de tres pliegues linguales abiertos en los molares inferiores.

En el Turoliense medio (MN12) de la Península Ibérica no se ha citado el hallazgo de Hystrix, a pesar de la existencia de yacimientos de esta edad muy ricos y diversos (por ejemplo Concud y Los Mansuetos). Este hecho, así como el registro de Hystrix primigenia en varias localidades españolas del Turoliense superior (MN13) hace pensar en una entrada de la especie en la Península Ibérica durante dicha edad, tal y como Mein et al. (1990) han señalado para la Fosa de Teruel.

\section{Lámina I}

Hystrix primigenia (Wagner, 1848) de Las Casiones. Escalas con divisiones de $1 \mathrm{~cm}$. La escala menor sólo para las figuras 1 y 2.

1-3 Hemimandíbula izquierda con $\mathrm{P}_{4}-\mathrm{M}_{3}$ (MNCN KS-122). 1 , vista labial. 2-3, vista oclusal.

4-6 $\mathrm{M}_{1 \cdot 2}$ izquierdo (MPZ-96/225 K93-1003). 4, vista oclusal. 5, vista labial. 6, vista lingual.
7 Serie superior derecha $\mathrm{P}^{4}-\mathrm{M}^{3}(\mathrm{MNCN} \mathrm{KS}-7)$ en vista oclusal.

8-10 $\mathrm{M}^{1-2}$ derecho (MPZ-96/226 K93-1078). 8, vista oclusal. 9 , vista lingual. 10 , vista labial.

$11 \mathrm{M}^{1-2}$ izquierdo (MNCN KS-1) en vista oclusal. 
Lámina I
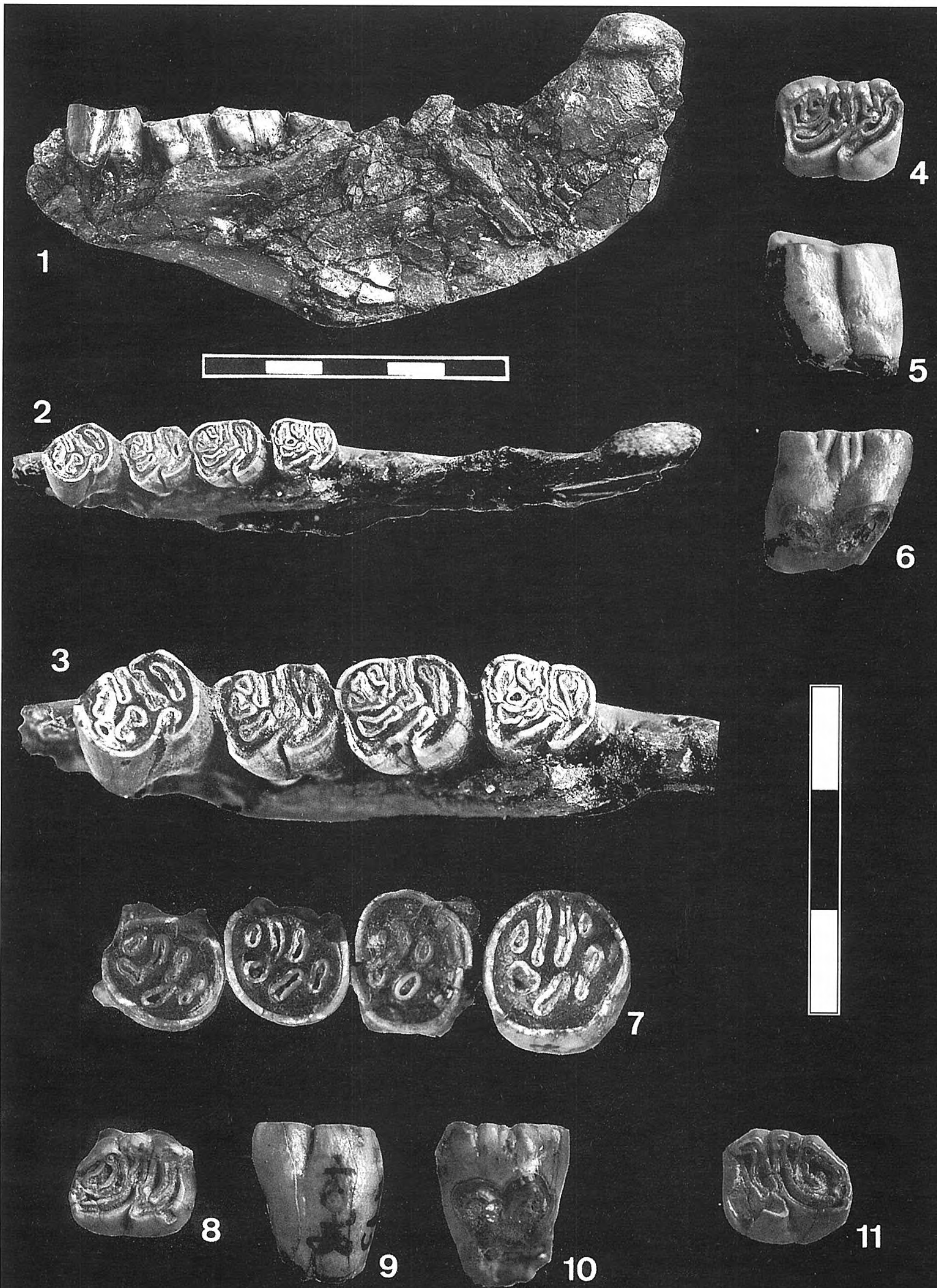


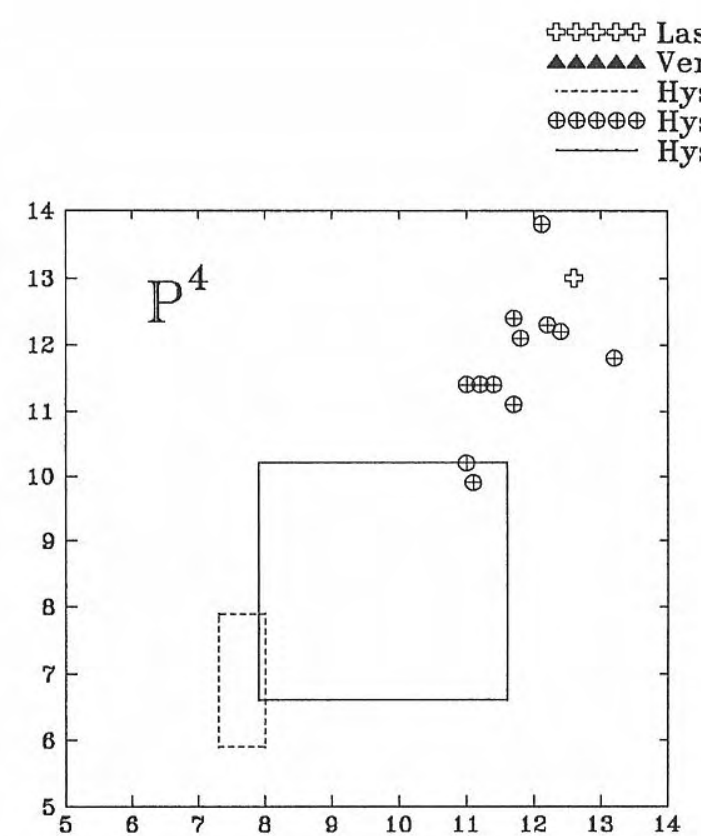

as Casiones

Venta del Moro

Hystrix parvae

strix primigenia

Hystrix refossa
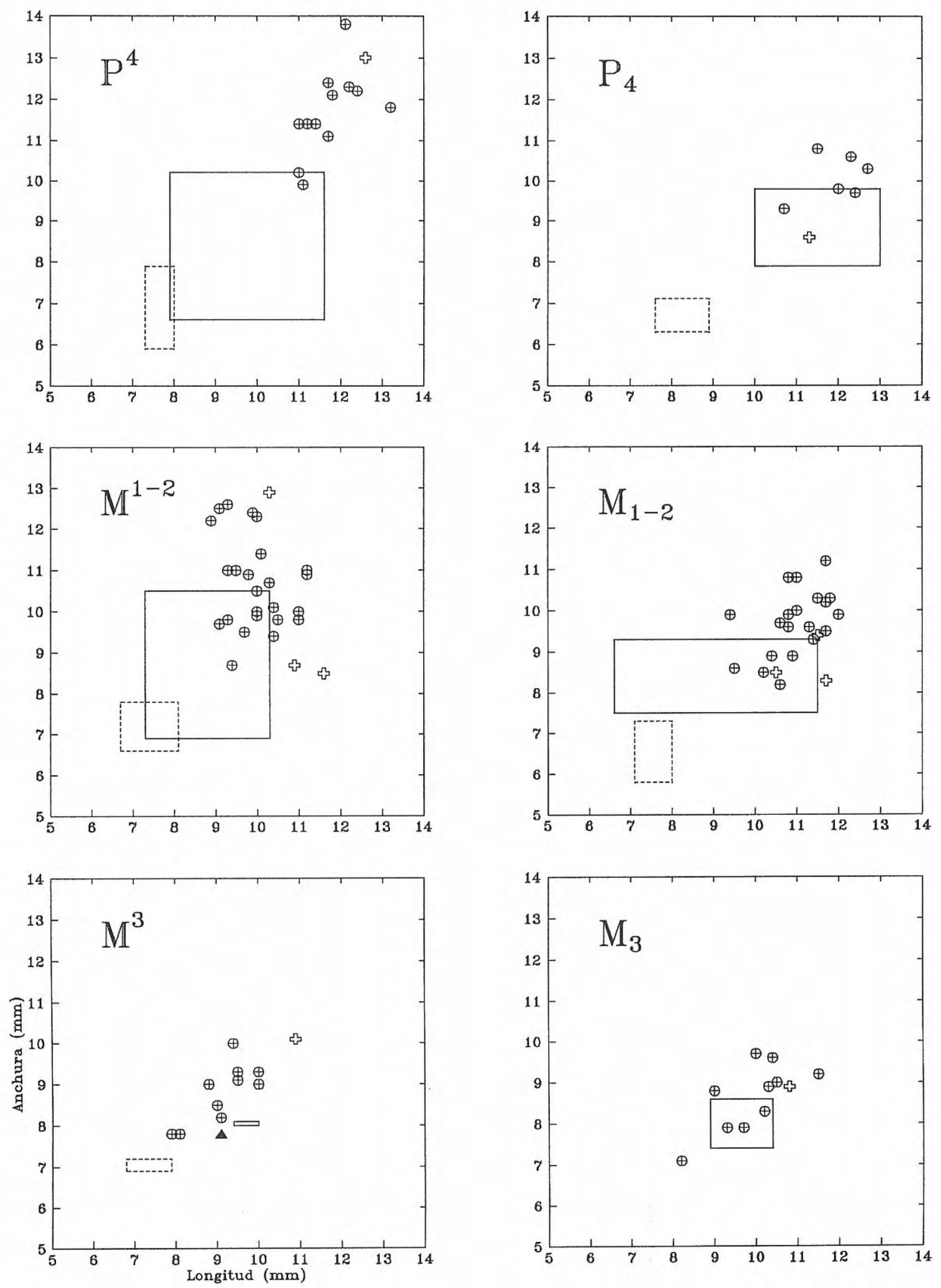

Figura 3. Diagramas de dispersión longitud-anchura de los dientes yugales de Hystrix de Las Casiones y Venta del Moro, Hystrix parvae (Kretzoi, 1951), Hystrix primigenia (Wagner, 1848) e Hystrix refossa Gervais, 1852. Datos tomados de Agustí et al. (1987), de Bonis et al. (1992), Koliadimou y Koufos (1991), Masini y Rook (1993), Montoya (1993), Sen (1994), Sen y Kovatchev (1987), Sulimski (1960), van Weers y Montoya (1996). 


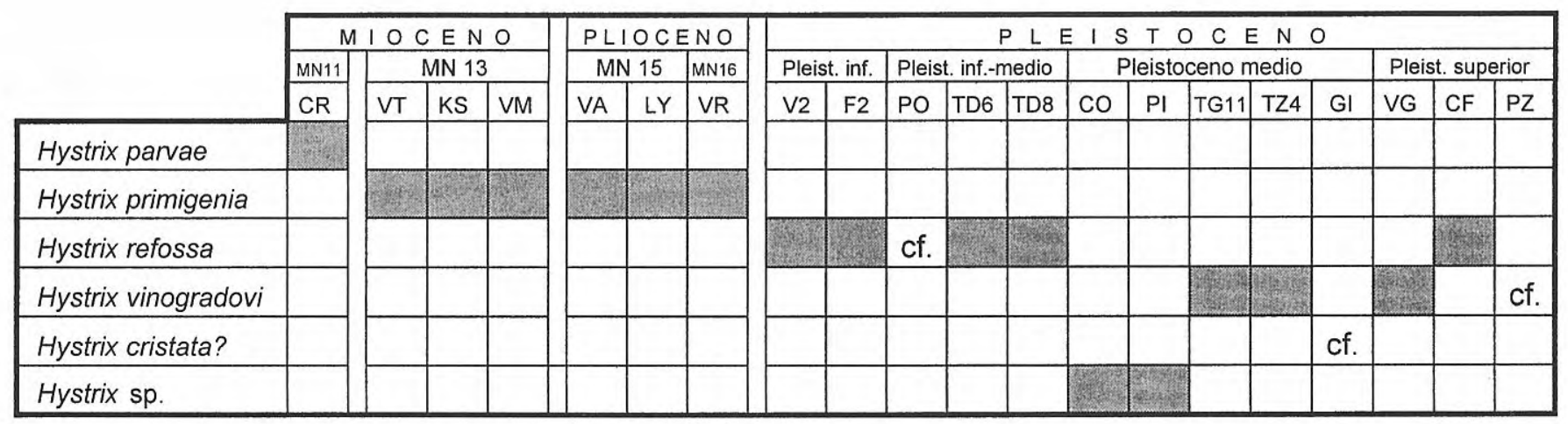

Figura 4. Distribución bioestratigráfica de Hystrix en España. CR: Crevillente 2, VT: Villastar, KS: Las Casiones, VM: Venta del Moro, VA: Villalba Alta, LY: Layna, VR: Villarroya, V2: Venta Micena 2, F2: Fuentenueva 2, PO: Pontón de la Oliva, TD6: Atapuerca TD6, TD8: Atapuerca TD8, CO: Cuevas del Congosto y de Las Figuras, PI: Pinilla del Valle, TG11: Atapuerca TG11, TZ4: Atapuerca TZ4, GI: Gibraltar, VG: Cueva de Valdegoba, CF: Castelldefels, PZ: Pedraza.

Aparte del hallazgo descrito en el presente trabajo, se ha encontrado también Hystrix en otros dos yacimientos españoles de la MN13: en Villastar (Teruel) y en Venta del Moro (Valencia). En Villastar se cita Hystrix primigenia (Mein et al., 1990; Adrover et al., 1993), pero no se describe ni figura el material. En Venta del Moro se ha encontrado escaso material, descrito y figurado en Morales (1984) y que se cita como Hystrix sp. Si efectuamos la comparación con los fósiles procedentes de otras localidades del Turoliense, se aprecia que el $\mathrm{M}^{3}$ de Venta del Moro presenta unas dimensiones (longitud: 9,1; anchura: 7,8) menores que las del diente homólogo de Pikermi (de Bonis et al., 1992), de Kalimanci (Sen y Kovatchev, 1987) y claramente inferiores a las del diente de Las Casiones, aquí estudiado; en cambio, su tamaño es muy similar al del $\mathrm{M}^{3}$ de $H$. primigenia de Dytiko 3 (de Bonis et al., 1992), siendo incluso mayor que los $\mathrm{M}^{3}$ de Kemiklitepe (Sen, 1994). Pensamos que el material de Venta del Moro puede asignarse sin problemas a Hystrix primigenia, en cuyo rango de talla encaja perfectamente (Fig. 3).

Ya en el Plioceno, se ha encontrado $H$. primigenia en varios yacimientos de la Península Ibérica. De Villalba Alta, Adrover (1986) figura un $\mathrm{M}^{\top}$ que presenta una morfología muy similar a los $\mathrm{M}^{1-2}$ de Las Casiones, aquí descritos (KS-1 y K93-1078). También se ha encontrado la especie en Layna y Villarroya (Agustí et al., 1987). Tanto Villalba Alta como Layna pertenecen a la zona MN15, pero Villarroya se sitúa en la MN16, según de Bruijn et al. (1992), lo que representa una de las citas más recientes en el registro fósil de la especie.

En cuanto a la presencia de Hystrix en el Pleistoceno español, la especie típica del Plioceno final y Cuaternario europeo, Hystrix refossa Gervais, 1852, se halla presente en distintas localidades del Pleistoceno inferior y medio, tales como Venta Micena 2, Fuentenueva 2, Pontón de la Oliva, Atapuerca y Castelldefels (Agustí et al., 1987; Sesé y Ruiz Bustos, 1992; Laplana y Cuenca, 1996), casi siempre citada como Hystrix major, especie que van Weers (1994) ha puesto en sinonimia con $H$. refossa. También se ha reconocido el pequeño Hystrix vinogradovi Argyropoulo, 1941, en Atapuerca y en la Cueva de Valdegoba (Díez et al., 1989; Sesé, 1994; Laplana y Cuenca, 1996), así como Hystrix cf. cristata? en Gibraltar. Por último, hay varias citas de Hystrix sp. en diversos yacimientos del Pleistoceno, como son Pinilla del Valle (Toni y Molero, 1990) y las cuevas del Congosto y de Las Figuras (Alberdi et al., 1977).

Por otra parte, en las colecciones del Museo Nacional de Ciencias Naturales se conserva una mandíbula inédita, con número de registro $\mathrm{MNCN}-2828$, procedente del Pleistoceno superior de Pedraza, en Segovia (Torres, 1969). Aunque carece de dentición yugal, la serie de alveolos $\mathrm{P}_{4}-\mathrm{M}_{3}$ mide $31 \mathrm{~mm}$ aproximadamente. Esta dimensión es claramente menor que en Hystrix refossa de Valdarno y Pirro Nord (Italia) y, en cambio, es muy semejante a la que presentan las mandíbulas de Hystrix vinogradovi del Pleistoceno del Cáucaso (ver van Weers, 1994). De todos modos, debido a la ausencia de la dentición yugal, conviene mantener cierta cautela; por ello creemos adecuado adscribir esta mandíbula a Hystrix cf. vinogradovi.

\section{AGRADECIMIENTOS}

Este trabajo se ha desarrollado en el marco de los proyectos de la DGICYT PB 92-0013 y PB 95-0114. La Diputación General de Aragón autorizó excavaciones en Las Casiones durante los años 1993 y 1994 (expedientes 040/93 y 064/94 del Departamento de Educación y Cultura). Tanto en el Museo Paleontológico de la Universidad de Zaragoza como en el Museo Nacional de Ciencias Naturales hemos encontrado todo tipo de facilidades para el estudio de los materiales. Agradecemos a David Herrero y familia su autorización para excavar en el terreno de su propiedad, así como el interés que mostraron hacia nuestro trabajo. También queremos mostrar nuestro agradecimiento a los doctores Nieves López y Remmert Daams, por la revisión del texto. 


\section{BIBLIOGRAFÍA}

Adrover, R. 1986. Nuevas faunas de roedores en el MioPlioceno continental de la región de Teruel (España). Interés bioestratigráfico y paleoecológico. Instituto de Estudios Turolenses (CSIC), Diputación Provincial de Teruel, 423 pp.

Adrover, R., Mein, P. y Moissenet, E. 1993. Roedores de la transición Mio-Plioceno de la región de Teruel. Paleontologia i Evolució, 26-27, 47-84.

Agustí, J., Arbiol, S. y Martín-Suárez, E. 1987. Roedores y lagomorfos (Mammalia) del Pleistoceno inferior de Venta Micena (depresión de Guadix-Baza, Granada). Paleontologia i Evolució, Mem. Esp. 1, 95-107.

Alberdi, M.T., Hoyos, M. y García, J.C. 1977. Estudio de la fauna y su situación en las Cuevas del Congosto y de Las Figuras, Alcorlo (Guadalajara). Speleon, 23, 103-119.

Alcalá, L. 1992. Macromamíferos neógenos de la fosa de Alfambra-Teruel. Tesis Doctoral, Univ. Complutense, Madrid, $521 \mathrm{pp}$.

Alcalá, L. 1994. Macromamíferos neógenos de la fosa de Alfambra-Teruel. Instituto de Estudios Turolenses Museo Nacional de Ciencias Naturales, Teruel, 554 pp.

Alcalá, L., Montoya, P. and Morales, J. 1994. New large mustelids from the Late Miocene of the Teruel Basin (Spain). Comptes Rendus de l'Academie des Sciences de Paris, 319, série II, 1093-1100.

Arambourg, C. 1959. Vertébrés continentaux du Miocène supérieur de l'Afrique du Nord. Publications Service Carte Géologique de l'Algérie, N.S., 4, 1-161.

Bachmayer, F. and Wilson, R.W. 1970. Small mammals (Insectivora, Chiroptera, Lagomorpha, Rodentia) from the Kohfidisch fissures of Burgenland, Austria. Annals Naturhistorischen Museum Wien, 74, 533-587.

Bonis, L. de, Bouvrain, G., Geraads, D. and Koufos, G.D. 1992. A skull of Hystrix primigenia from the late Miocene of Macedonia (Greece). Neues Jahrbuch für Geologie und Paläontologie Monatshefte, 2, 75-87.

Bruijn, H. de, Daams, R., Daxner-Höck, G., Fahlbusch, V., Ginsburg, L., Mein, P. and Morales, J. 1992. Report of the RCMNS working group on fossil mammals, Reisensburg 1990. Newsletter Stratigraphy, 26, 65-118.

Cerdeño, E. 1989. Revisión de la sistemática de los rinocerontes del Neógeno de España. Tesis Doctoral, Universidad Complutense de Madrid, 306/89, 429 pp.

Cerdeño, E. 1990. Los rinocerótidos del Mioceno superior de España. Paleontologia i Evolució, 23, 47-53.

Depéret, C. 1890-1892. Les animaux pliocènes du Roussillon. Mémoires Societé Géologique France, 3, 1194.

Díez, C., García, M.A., Gil, E., Jordá, J.F., Ortega, A.I., Sánchez, A. y Sánchez, B. 1989. La Cueva de Valdegoba (Burgos). Primera campaña de excavaciones. Zephyrus, 61-62, 55-74.

Garevski, R. 1956. Neue Fundstellen der Pikermifauna in Mazedonien. Acta Musei Macedonici Scientiarum Naturalium, 4, 69-96.

Gaudry, A. 1862-1867. Animaux fossiles et Géologie de l'Attique. F. Savy (Ed.), Societé Géologique de France, $474 \mathrm{pp}$.
Koliadimou, K. and Koufos, G.D. 1991. The Hystricidae from the Pleistocene of Macedonia (Greece) and a review of the European representatives of the family. Bulletin of the Geological Society of Greece, 25, 453471.

Kretzoi, M. 1951. The Hipparion Fauna from Csákvár. Földtani Közlöny, 81, 384-417.

Laplana, C. y Cuenca, G. 1996. Hystricidae (Rodentia, Mammalia) del Pleistoceno de la Sierra de Atapuerca (Burgos, España). Resúmenes XII Bienal de la Real Sociedad Española de Historia Natural, 194.

Masini, F. and Rook, L. 1993. Hystrix primigenia (Mammalia, Rodentia) from the Late Messinian of the Monticino gypsum quarry (Faenza, Italy). Bolletino della Società Paleontologica Italiana, 32 (1), 79-87.

Mein, P., Moissenet, E. et Adrover, R. 1990. Biostratigraphie du Néogène Supérieur du bassin de Teruel. Paleontologia i Evolució, 23, 121-139.

Montoya, P. 1993. The porcupine Hystrix suevica Schlosser, 1884 from the Lower Turolian of Crevillente 2 (Spain). Scripta Geologica, 103, 135-149.

Montoya, P. 1994. Los macromamíferos del Mioceno superior del área de Crevillente (Alicante). Tesis Doctoral, Universitat de València, $421 \mathrm{pp}$.

Morales, J. 1984. Venta del Moro, su macrofauna de mamíferos y biostratigrafía continental del Mioceno terminal mediterráneo. Ed. Univ. Complutense, Madrid, 340 pp.

Nikiforowa, K.W. y Vangengeim, E.A. 1988. [Bioestratigrafía del Plioceno Superior-Pleistoceno Inferior de Tadzjikistan] (en ruso). Akademia Nauk U.R.S.S., Moscú, 1-125.

Ozansoy, F. 1965. Etude des gisements continentaux et des Mammifères du Cénozoique de Turquie. Mémoires Societé Géologique France, 44, 1-92.

Schlosser, M. 1884. Die Nager der europäischen tertiärs nebst Betrachtungen über die Organisation und die geschichtliche Entwicklung der Nager überhaupt. Palaeontographica, 31, 3, F.7, 19-162.

Schlosser, M. 1902. Beiträge zur Kenntnis der Säugethierreste auf den süddeutschen Bohnerzen. Geologie und Paläontologie Abhandlung, n.f., Bd. 5, H. 3, 115-258.

Sen, S. 1994. Les gisements de mammifères du Miocène supérieur de Kemiklitepe, Turquie, 5. Rongeurs, Tubulidentés et Chalicothères. Bulletin du Muséum National d'Histoire Naturelle, 4' Sér., sec. C, 16 (1), 97111.

Sen, S. and Kovatchev, D.B. 1987. The porcupine Hystrix primigenia (Wagner) from the Late Miocene of Bulgaria. Proceedings Koninklijke Nederlandse Akademie Van Wetenschappen, B, 90 (4), 317-323.

Sesé, C. 1994. Paleoclimatical interpretation of the Quaternary small mammals of Spain. Geobios, 27, 753767.

Sesé, C. y Ruiz Bustos, A. 1992. Nuevas faunas de Micromamíferos del Pleistoceno del Norte de la Provincia de Madrid (España). Boletín de la Real Sociedad Española de Historia Natural (Sec. Geol.), 87, 115-139. 
Solounias, N. 1981. Mammalian fossils of Samos and Pikermi. Part 2. Resurrection of a classic Turolian fauna. Annals of Carnegie Museum, 50, 231-270.

Sulimski, A. 1960. Hystrix primigenia (Wagner) in the Pliocene fauna from Weze. Acta Palaeontologica Polonica, 5 (3), 319-336.

Thomas, H. et Petter, G. 1986. Révision de la faune de mammifères du Miocène supérieur de Menacer (exMarceau), Algérie, discussion sur l'âge du gisement. Geobios, 19, 357-373.

Toni, I. y Molero, G. 1990. Los roedores (Rodentia, Mammalia) del yacimiento cuaternario de Pinilla del Valle (Madrid). In Actas de Paleontología (Eds. J. Civis y J.A. Flores). Univ. Salamanca, 359-373.

Torres, T. J. de. 1969. Un hiénido de las cavernas de Pedraza de la Sierra (Segovia). Boletín Geológico y Minero, 80, 231-234.

Wagner, A. 1848. Urweltliche Saugethierreste aus Griechenland. Abhandlungen Bayerische Akademie
Wissenschaf, 5, 333-378.

Weers, D.J. van. 1990. Dimensions and occlusal patterns in molars of Hystrix brachyura Linnaeus, 1758 (Mammalia, Rodentia) in a system of wear categories. Bijdragen tot de Dierkunde, 60, 121-134.

Weers, D.J. van. 1993. Teeth morphology and taxonomy of the Miocene rodent Anchitheriomys suevicus (Schlosser, 1884), with notes on the family Hystricidae. Proceedings Koninklijke Nederlandse Akademie Van Wetenschappen, 96, 81-89.

Weers, D.J. van. 1994. The porcupine Hystrix refossa Gervais, 1852 from the Plio-Pleistocene of Europe, with notes on other fossil and extant species of the genus Hystrix. Scripta Geologica, 106, 35-52.

Weers, D.J. van and Montoya, P. 1996. Taxonomy and stratigraphic record of the oldest European porcupine Hystrix parvae (Kretzoi, 1951). Proceedings Koninklijke Nederlandse Akademie van Wetenschappen, 99, 131141. 